\title{
Eventually Periodic Solutions of a Max-Type Difference Equation
}

\author{
Taixiang Sun, ${ }^{1}$ Jing Liu, ${ }^{1}$ Qiuli He, ${ }^{2}$ Xin-He Liu, ${ }^{1}$ and Chunyan Tao ${ }^{1}$ \\ ${ }^{1}$ College of Mathematics and Information Science, Guangxi University, Nanning, Guangxi 530004, China \\ ${ }^{2}$ College of Electrical Engineering, Guangxi University, Nanning, Guangxi 530004, China \\ Correspondence should be addressed to Taixiang Sun; stx1963@163.com
}

Received 25 April 2014; Accepted 19 June 2014; Published 1 July 2014

Academic Editor: Baojian Hong

Copyright (c) 2014 Taixiang Sun et al. This is an open access article distributed under the Creative Commons Attribution License, which permits unrestricted use, distribution, and reproduction in any medium, provided the original work is properly cited.

\begin{abstract}
We study the following max-type difference equation $x_{n}=\max \left\{A_{n} / x_{n-r}, x_{n-k}\right\}, n=1,2, \ldots$, where $\left\{A_{n}\right\}_{n=1}^{+\infty}$ is a periodic sequence with period $p$ and $k, r \in\{1,2, \ldots\}$ with $\operatorname{gcd}(k, r)=1$ and $k \neq r$, and the initial conditions $x_{1-d}, x_{2-d}, \ldots, x_{0}$ are real numbers with $d=\max \{r, k\}$. We show that if $p=1$ (or $p \geq 2$ and $k$ is odd), then every well-defined solution of this equation is eventually periodic with period $k$, which generalizes the results of (Elsayed and Stević (2009), Iričanin and Elsayed (2010), Qin et al. (2012), and Xiao and Shi (2013)) to the general case. Besides, we construct an example with $p \geq 2$ and $k$ being even which has a well-defined solution that is not eventually periodic.
\end{abstract}

\section{Introduction}

The max operator arises naturally in certain models in automatic control theory (see [1]). In recent years, the discrete case involving difference equations with maximum has been receiving increasing attention, for some results in this area; see, for example, [2-4].

In this paper, we consider the following max-type equation:

$$
x_{n}=\max \left\{\frac{A_{n}}{x_{n-r}}, x_{n-k}\right\}, \quad n=1,2, \ldots,
$$

where $\left\{A_{n}\right\}_{n=1}^{+\infty}$ is a periodic sequence with period $p$ and $k, r \in\{1,2, \ldots\}$ with $\operatorname{gcd}(k, r)=1$ and $k \neq r$, and the initial conditions $x_{1-d}, x_{2-d}, \ldots, x_{0}$ are real numbers with $d=$ $\max \{r, k\}$.

In [5], Iričanin and Elsayed showed that every welldefined solution of (1) is eventually periodic with period 4 when $k=4, r=1$, and $p=1$. Elsayed and Stević [6] showed that every well-defined solution of (1) is eventually periodic with period 3 when $k=3, r=1$, and $p=1$. In [7], Xiao and Shi showed that if $k=2, r=1$, and $p=1$, then every well-defined solution of (1) is eventually periodic with period
2. Qin et al. [8] showed that every well-defined solution of (1) is eventually periodic with period $k$ when $r=1$ and $p=1$.

In this paper, we will generalize the results of [5-8] to the general case.

\section{Main Results and Example}

In this section, we are ready to state and prove the main results.

Theorem 1. Let $\left\{A_{n}\right\}_{n=1}^{+\infty}$ be a periodic sequence with period $p$, and $k, r \in\{1,2, \ldots\}$ with $g c d(k, r)=1$ and $k \neq r$.

(1) If $p \geq 2$ and $k$ is odd, then every well-defined solution of (1) is eventually periodic with period $k$.

(2) If $p=1$, then every well-defined solution of (1) is eventually periodic with period $k$.

Proof. Let $\left\{x_{n}\right\}_{n=1-d}^{+\infty}$ be a well-defined solution of (1). It follows from (1) that, for any $n \geq 0$ and any $i \geq 0$,

$$
x_{(n+1) k+i}=\max \left\{\frac{A_{(n+1) k+i}}{x_{(n+1) k+i-r}}, x_{n k+i}\right\} \geq x_{n k+i} .
$$


Then, for every $i \geq 0,\left\{x_{n k+i}\right\}_{n=0}^{+\infty}$ is increasing, and $x_{n k+i}<0$ for all $n \geq 0$ or there exists $N_{i}>0$ such that $x_{n k+i}>0$ for all $n \geq N_{i}$.

We claim that $\left\{x_{n k}\right\}_{n=0}^{+\infty}$ is a constant sequence eventually. Indeed, if $\left\{x_{n k}\right\}_{n=0}^{+\infty}$ is not constant sequence eventually, then there exist $k r<n_{1}<n_{2}<\cdots$ such that $x_{n_{i} k}>x_{\left(n_{i}-1\right) k}$ and $A_{n_{i} k}$ is a constant sequence for all $i \geq 1$ since $\left\{A_{n}\right\}_{n=1}^{+\infty}$ is a periodic sequence. Thus we have

$$
\begin{aligned}
x_{n_{i+1} k} & =\max \left\{\frac{A_{n_{i+1} k}}{x_{n_{i+1} k-r}}, x_{\left(n_{i+1}-1\right) k}\right\} \\
& =\frac{A_{n_{i+1} k}}{x_{n_{i+1} k-r}}>x_{\left(n_{i+1}-1\right) k} \\
& \geq x_{n_{i} k}=\max \left\{\frac{A_{n_{i} k}}{x_{n_{i} k-r}}, x_{\left(n_{i}-1\right) k}\right\} \\
& =\frac{A_{n_{i} k}}{x_{n_{i} k-r}} .
\end{aligned}
$$

From this we obtain that, for all $i \geq 1$,

$$
\frac{A_{n_{i} k}\left(x_{n_{i+1} k-r}-x_{n_{i} k-r}\right)}{x_{n_{i+1} k-r} x_{n_{i} k-r}}<0 .
$$

It follows that, for all $i \geq 1$,

$$
x_{n_{i} k} x_{n_{i} k-r}=A_{n_{i} k}<0, \quad x_{n_{i+1} k-r}>x_{n_{i} k-r} .
$$

Therefore we have $x_{n k} x_{n k-r}<0$ eventually. By induction, we can show that $x_{n k-i r} x_{n k-(i+1) r}<0$ eventually for all $1 \leq i \leq$ $k-1$ and every $\left\{x_{n k-i r}\right\}_{n=r}^{+\infty} \quad(1 \leq i \leq k-1)$ is not constant sequence eventually.

If $p \geq 2$ and $k$ is odd, then we have $x_{n k} x_{n k-k r} \prod_{i=1}^{k-1} x_{n k-i r} x_{n k-i r}<0$ eventually. This is a contradiction.

If $p=1$, then we write $A_{n}=A$ for all $n \geq 1$ and choose $m_{0} \geq m_{1} \geq \cdots \geq m_{2 k}$ such that $x_{m_{j} k-j r}<x_{\left(m_{j}+1\right) k-j r}$ and $x_{\left(m_{j}+1\right) k-(j+1) r}=x_{\left(m_{j+1}+1\right) k-(j+1) r}$ for any $j \in\{0,1, \ldots, 2 k-1\}$. Thus

$$
\begin{aligned}
x_{\left(m_{0}+1\right) k} & =\max \left\{\frac{A}{x_{\left(m_{0}+1\right) k-r}}, x_{m_{0} k}\right\} \\
& =\frac{A}{x_{\left(m_{0}+1\right) k-r}} \\
& =\frac{A}{x_{\left(m_{1}+1\right) k-r}} \\
& =\frac{A}{\max \left\{A / x_{\left(m_{1}+1\right) k-2 r}, x_{m_{1} k-r}\right\}}
\end{aligned}
$$

$$
\begin{aligned}
& =x_{\left(m_{1}+1\right) k-2 r} \\
& =x_{\left(m_{2}+1\right) k-2 r} \\
& \quad \vdots \\
& =x_{\left(m_{2 k}+1\right) k-2 k r} \\
& \leq x_{m_{0} k},
\end{aligned}
$$

which is a contradiction. This completes the proof of the claim.

By the above claim we may choose an $N>0$ such that $x_{n k}=x_{N k}$ for all $n \geq N$. Since $A_{n}$ is a periodic sequence, we can choose an $N_{1}>N$ such that $A_{N_{1} k+r} / x_{N k}=$ $\max \left\{A_{n k+r} / x_{N k}: n \geq N\right\}$. Then, for all $n \geq N_{1}$,

$$
x_{n k+r}=\max \left\{\frac{A_{n k+r}}{x_{N k}}, x_{(n-1) k+r}\right\} .
$$

Thus $x_{n k+r}=x_{(n-1) k+r}$ for all $n \geq N_{1}+1$ (otherwise, if $x_{m k+r}>x_{(m-1) k+r}$ for some $m \geq N_{1}+1$, then we have $x_{m k+r}=A_{m k+r} / x_{N k}>x_{(m-1) k+r} \geq x_{N_{1} k+r} \geq A_{N_{1} k+r} / x_{N k}$, which is a contradiction). By induction, we can show that $x_{n k+j r}$ is a constant sequence eventually for every $1 \leq j \leq k$. Note $\{j r: 1 \leq j \leq k\}_{\bmod k}=\{0,1,2, \ldots, k-1\}$ since $\operatorname{gcd}(k, r)=1$. Then $x_{n k+i}$ is a constant sequence eventually for every $i \in\{0,1, \ldots, k-1\}$, which implies that $\left\{x_{n}\right\}_{n=1-d}^{+\infty}$ is eventually periodic with period $k$.

From the proof of Theorem 1 we obtain the following corollary.

Corollary 2. Let $k, r \in\{1,2, \ldots\}$ with $k \neq r$. If $\left\{A_{n}\right\}_{n=1}^{+\infty}$ is a periodic sequence, then every positive (or negative) solution of (1) is eventually periodic with period $k$.

Theorem 3. Let $\left\{A_{n}\right\}_{n=1}^{+\infty}$ be a periodic sequence with period $p \geq 2$, and $k, r \in\{1,2, \ldots\}$ with $\operatorname{gcd}(k, r)=1$ and $k \neq r$. If $A_{s} \geq 0$ for some $s \in\{1,2, \ldots, p\}$, then every well-defined solution of (1) is eventually periodic with period $k$.

Proof. Let $\left\{x_{n}\right\}_{n=1-d}^{+\infty}$ be a well-defined solution of (1). Using arguments similar to the ones developed in the proof of Theorem 1, we know that, for every $i \geq 0,\left\{x_{n k+i}\right\}_{n=0}^{+\infty}$ is increasing, and $x_{n k+i}<0$ for all $n \geq 0$ or there exists $N_{i}>0$ such that $x_{n k+i}>0$ for all $n \geq N_{i}$.

We may assume without loss of generality that $A_{1} \geq 0$. We claim that $\left\{x_{n k}\right\}_{n=0}^{+\infty}$ is a constant sequence eventually. Indeed, if $\left\{x_{n k}\right\}_{n=0}^{+\infty}$ is not constant sequence eventually, then there exist $k r<n_{1}<n_{2}<\cdots$ such that $x_{n_{i} k}>x_{\left(n_{i}-1\right) k}$ with $A_{n_{i} k}$ being a constant sequence for all $i \geq 1$. Thus we have

$$
\begin{aligned}
x_{n_{i+1} k} & =\max \left\{\frac{A_{n_{i+1} k}}{x_{n_{i+1} k-r}}, x_{\left(n_{i+1}-1\right) k}\right\} \\
& =\frac{A_{n_{i+1} k}}{x_{n_{i+1} k-r}}>x_{\left(n_{i+1}-1\right) k}
\end{aligned}
$$




$$
\begin{gathered}
\geq x_{n_{i} k}=\max \left\{\frac{A_{n_{i} k}}{x_{n_{i} k-r}}, x_{\left(n_{i}-1\right) k}\right\} \\
=\frac{A_{n_{i} k}}{x_{n_{i} k-r}}, \\
x_{n p k}=\max \left\{\frac{A_{n p k}}{x_{n p k-r}}, x_{(n p-1) k}\right\} \geq \frac{A_{n p k}}{x_{n p k-r}} .
\end{gathered}
$$

From this we obtain that, for all $i \geq 1$,

$$
\begin{gathered}
x_{n_{i} k} x_{n_{i} k-r}=A_{n_{i} k}, \\
x_{n p k} x_{n p k-r} \geq A_{1} \geq 0 .
\end{gathered}
$$

Thus $A_{n_{i} k} \geq 0$ and

$$
\frac{A_{n_{i} k}\left(x_{n_{i+1} k-r}-x_{n_{i} k-r}\right)}{x_{n_{i+1} k-r} x_{n_{i} k-r}}<0 .
$$

It follows that, for all $i \geq 1$,

$$
x_{n_{i+1} k-r}<x_{n_{i} k-r} .
$$

This is a contradiction.

Using arguments similar to the ones developed in the proof of Theorem 1, we can show that $x_{n k+j r}$ is a constant sequence eventually for every $1 \leq j \leq k$. Note $\{j r: 1 \leq j \leq$ $k\}_{\bmod k}=\{0,1,2, \ldots, k-1\}$ since $\operatorname{gcd}(k, r)=1$. Then $x_{n k+i}$ is a constant sequence eventually for every $i \in\{0,1, \ldots, k-$ $1\}$, which implies that $\left\{x_{n}\right\}_{n=1-d}^{+\infty}$ is eventually periodic with period $k$.

Now we construct an example with $p \geq 2$ and $k$ being even which has a well-defined solution that is not eventually periodic.

Example 4. Consider the max-type equation

$$
x_{n}=\max \left\{\frac{A_{n}}{x_{n-r}}, x_{n-k}\right\}, \quad n=1,2, \ldots,
$$

where $k, r \in\{1,2, \ldots\}$ and $k$ is even with $\operatorname{gcd}(k, r)=1$ and $k \neq r$ and $A_{n}$ is a periodic sequence with $A_{2 i}=A<A_{2 i-1}=$ $B<0$ for all $i \geq 1$. Choose the initial conditions $x_{-i}=B$ for odd $i \in\{0,1, \ldots, d\}$ and $x_{-i}=1$ for even $i \in\{0,1, \ldots, d\}$ with $d=\max \{r, k\}$; we can obtain a solution $\left\{x_{n}\right\}_{n=-1}^{\infty}$ of (12) such that

(1) If $r<k$, then

$$
x_{n k+i}= \begin{cases}\left\{\frac{B}{A}\right\}^{n} B, & \text { if } i \in\{1,3, \ldots, r\}, \\ \left\{\frac{B}{A}\right\}^{n+1} B, & \text { if } i \in\{r+2, \ldots, k-1\}, \\ \left\{\frac{A}{B}\right\}^{n+1}, & \text { if } i \in\{2, \ldots, k\} .\end{cases}
$$

It is easy to verify that $\lim _{n \rightarrow \infty} x_{2 n}=\infty$ and $\lim _{n \rightarrow \infty} x_{2 n-1}=$ 0 .

$$
\text { (2) If } r>k \text {, then }
$$

$$
x_{n 2 r+i}= \begin{cases}\left\{\frac{B}{A}\right\}^{n} B, & \text { if } i \in\{1,3, \ldots, r\}, \\ \left\{\frac{B}{A}\right\}^{n+1} B, & \text { if } i \in\{r+2, \ldots, 2 r-1\}, \\ \left\{\frac{A}{B}\right\}^{n+1}, & \text { if } i \in\{2, \ldots, 2 r\} .\end{cases}
$$

It is easy to verify that $\lim _{n \rightarrow \infty} x_{2 n}=\infty$ and $\lim _{i \rightarrow \infty} x_{2 n-1}=$ 0 .

Remark 5. Consider the max-type equation

$$
x_{n}=\max \left\{\frac{A_{n}}{x_{n-s r}}, x_{n-s k}\right\}, \quad n=1,2, \ldots,
$$

where $\left\{A_{n}\right\}_{n=1}^{+\infty}$ is a periodic sequence with period $p s$ and $s, k, r \in\{1,2, \ldots\}$ with $\operatorname{gcd}(k, r)=1$ and $k \neq r$, and $p=1$ (or $p \geq 2$ ), and the initial conditions $x_{1-d}, x_{2-d}, \ldots, x_{0}$ are real numbers with $d=\max \{s r, s k\}$. Write $y_{n}^{i}=x_{n s+i}$ for every $1 \leq i \leq s$ and $n=0,1,2, \ldots$. Then (12) reduces to the equation

$$
y_{n}^{i}=\max \left\{\frac{A_{n s+i}}{y_{n-r}^{i}}, y_{n-k}^{i}\right\}, \quad 1 \leq i \leq s, n=0,1,2, \ldots
$$

(1) If $p=1$ (or $p \geq 2$ and $k$ is odd), then it follows from Theorem 1 that, for every $1 \leq i \leq s$, every well-defined solution of equation $y_{n}^{i}=\max \left\{A_{n s+i} / y_{n-r}^{i}, y_{n-k}^{i}\right\}$ is eventually periodic with period $k$. Thus every welldefined solution of (15) is eventually periodic with period $s k$.

(2) If $p \geq 2$ and, for every $1 \leq i \leq s$, there exists some $j_{i}$ such that $A_{j_{i} s+i} \geq 0$, then it follows from Theorem 3 that for every $1 \leq i \leq s$, every well-defined solution of equation $y_{n}^{i}=\max \left\{A_{n s+i} / y_{n-r}^{i}, y_{n-k}^{i}\right\}$ is eventually periodic with period $k$. Thus every welldefined solution of (15) is eventually periodic with period $s k$.

(3) If $p \geq 2$ and $k$ is even, then it follows from Example 4 that, for every $1 \leq i \leq s$, we can construct an equation $y_{n}^{i}=\max \left\{A_{n s+i} / y_{n-r}^{i}, y_{n-k}^{i}\right\}$ such that it has a welldefined solution which is not eventually periodic. Thus we can construct an equation

$$
x_{n}=\max \left\{\frac{A_{n}}{x_{n-s r}}, x_{n-s k}\right\}, \quad n=1,2, \ldots
$$

such that it has a well-defined solution which is not eventually periodic.

\section{Conflict of Interests}

The authors declare that there is no conflict of interests regarding the publication of this paper. 


\section{Acknowledgments}

The project is supported by NNSF of China (11261005) and NSF of Guangxi (2012GXNSFDA276040).

\section{References}

[1] E. P. Popov, Automatic Regulation and Control, Nauka, Moscow, Russia, 1966, (Russian).

[2] A. Gelişken and C. Çinar, "On the global attractivity of a maxtype difference equation," Discrete Dynamics in Nature and Society, vol. 2009, Article ID 812674, 5 pages, 2009.

[3] W. Liu and X. Yang, "Quantitative bounds for positive solutions of a Stević difference equation," Discrete Dynamics in Nature and Society, vol. 2010, Article ID 235808, 14 pages, 2010.

[4] T. Sun, B. Qin, H. Xi, and C. Han, "Global behavior of the maxtype difference equation $x_{n+1}=\max \left\{1 / x_{n}, A_{n} / x_{n}-1\right\}$," Abstract and Applied Analysis, vol. 2009, Article ID 152964, 10 pages, 2009.

[5] B. D. Iričanin and E. M. Elsayed, "On the max-type difference equation $x_{n+1}=\max \left\{A / x_{n}, x_{n-3}\right\}$," Discrete Dynamics in Nature and Society, vol. 2010, Article ID 675413, 13 pages, 2010.

[6] E. M. Elsayed and S. Stević, "On the max-type equation $x_{n+1}=$ $\max \left\{A / x_{n}, x_{n-2}\right\}$," Nonlinear Analysis: Theory, Methods and Applications, vol. 71, no. 3-4, pp. 910-922, 2009.

[7] Q. Xiao and Q. Shi, "Eventually periodic solutions of a max-type equation," Mathematical and Computer Modelling, vol. 57, no. 34, pp. 992-996, 2013.

[8] B. Qin, T. Sun, and H. Xi, "Dynamics of the max-type difference equation $x_{n+1}=\max \left\{A / x_{n}, x_{n-k}\right\}$," Journal of Computational Analysis and Applications, vol. 14, pp. 856-861, 2012. 


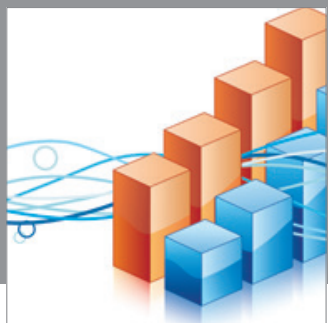

Advances in

Operations Research

mansans

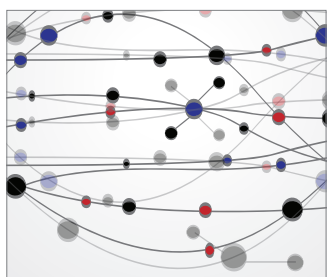

The Scientific World Journal
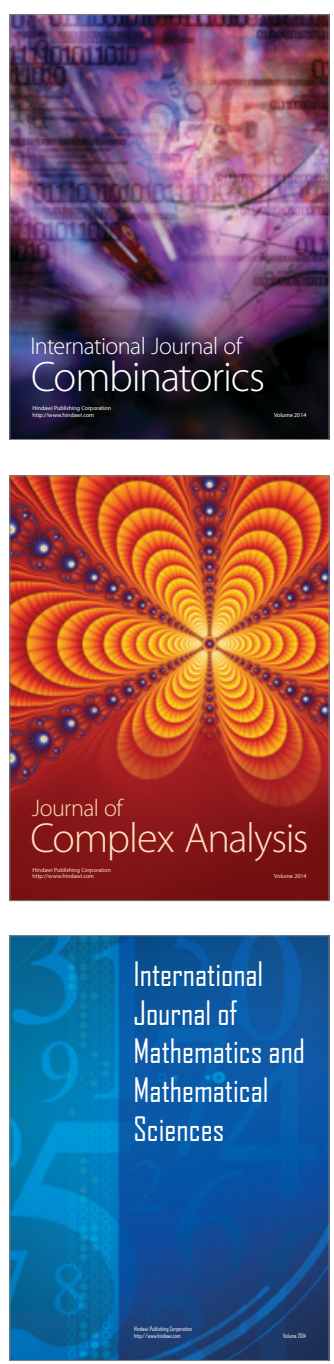
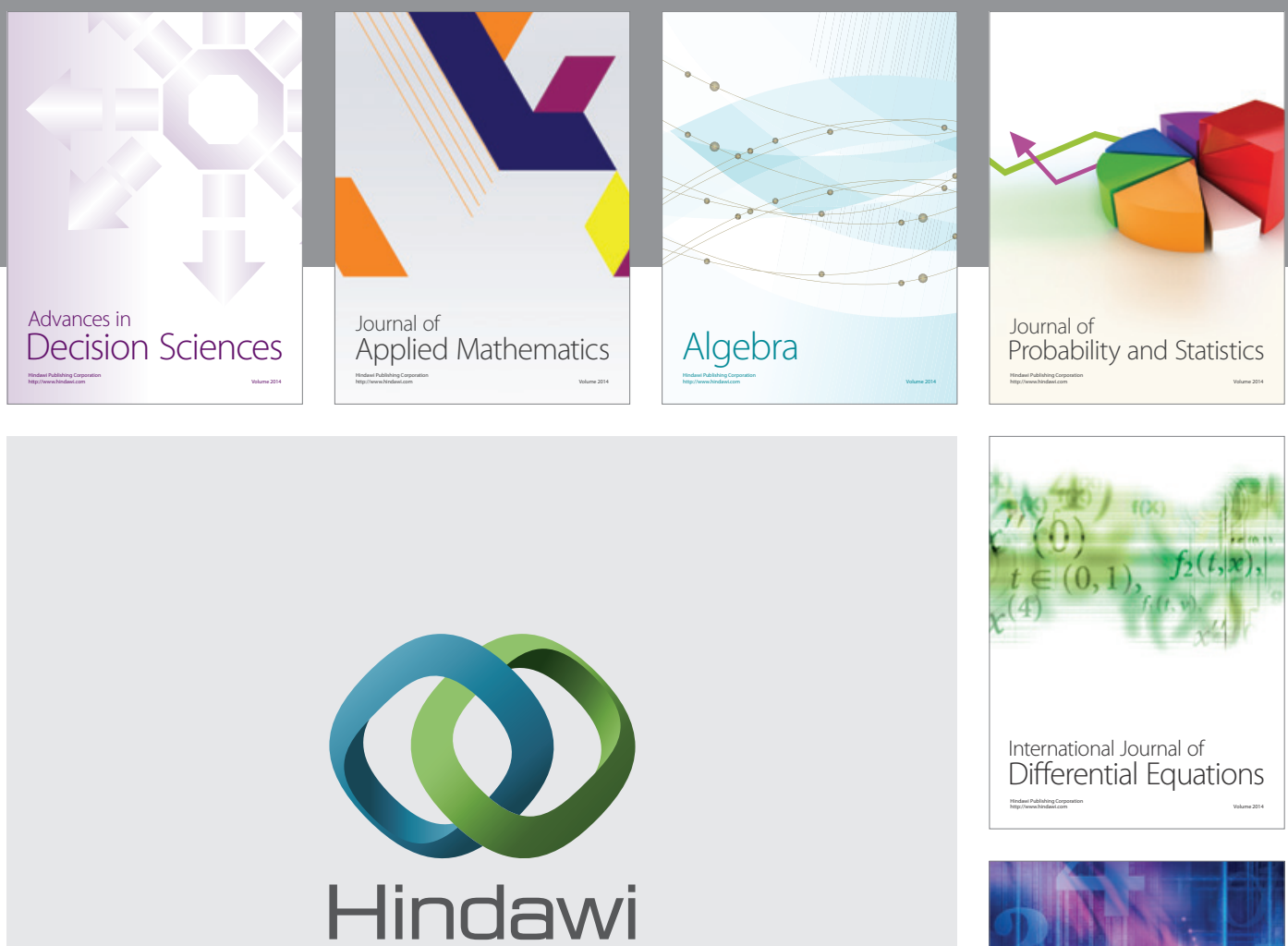

Submit your manuscripts at http://www.hindawi.com
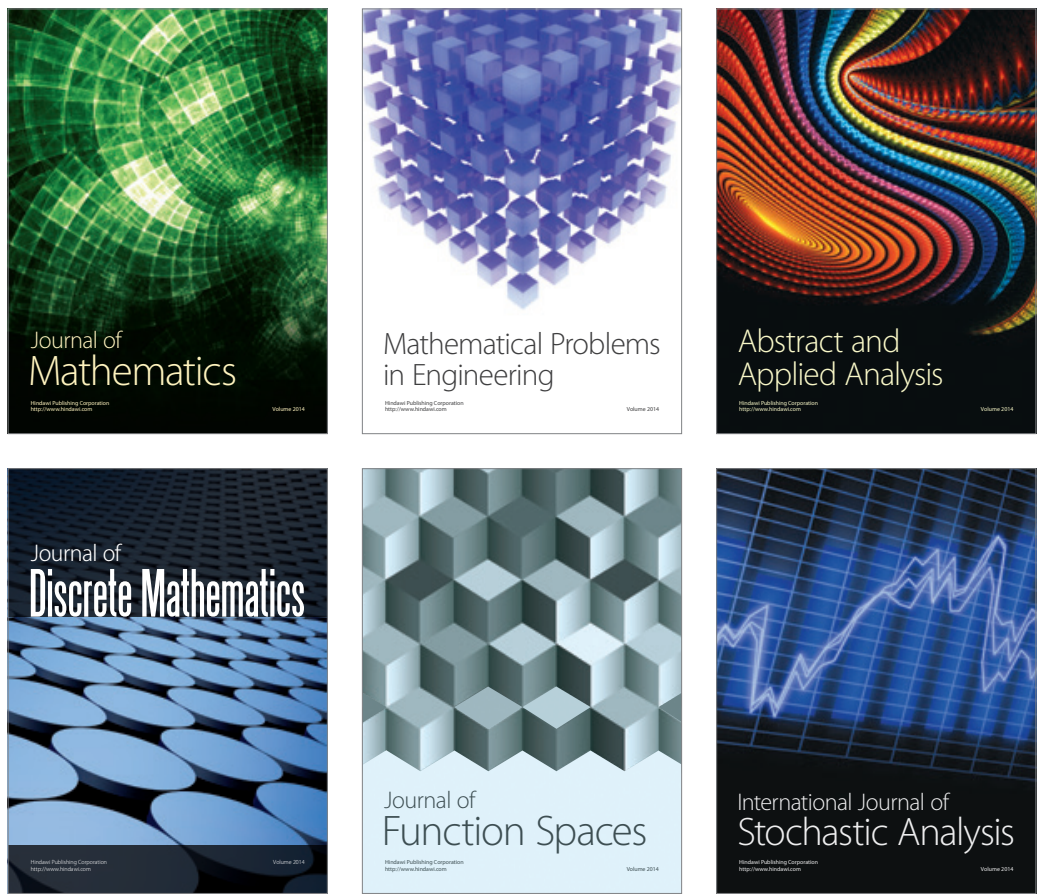

Journal of

Function Spaces

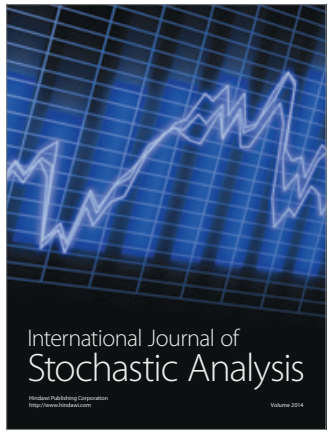

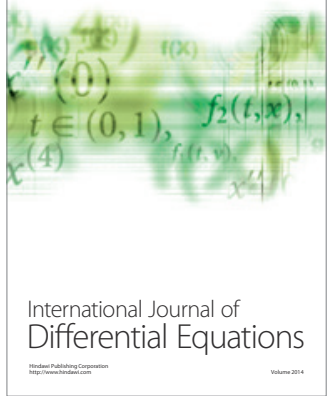
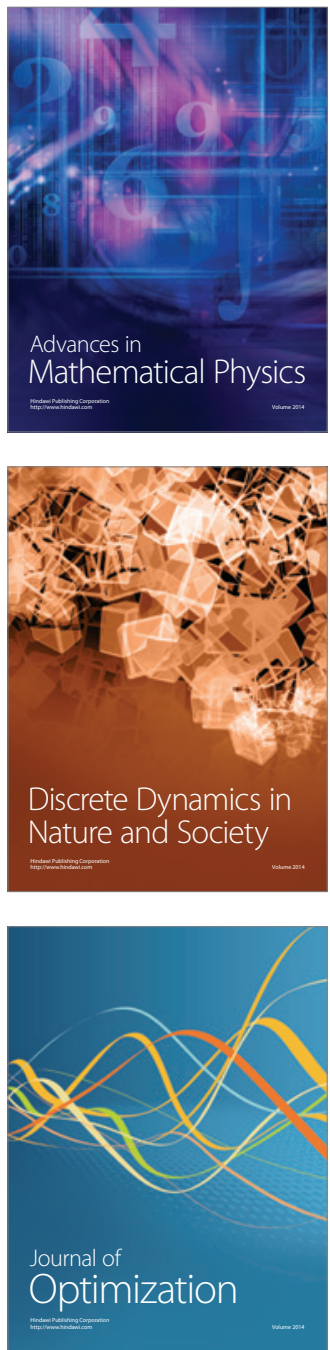